\title{
Applying cultural evolution to sustainability challenges: an introduction to the special issue
}

\author{
Jeremy S. Brooks ${ }^{1} \cdot$ Timothy M. Waring ${ }^{2} \cdot$ Monique Borgerhoff Mulder $^{3} \cdot$ Peter J. Richerson $^{4}$
}

Received: 22 November 2017 / Accepted: 27 November 2017 / Published online: 14 December 2017

(c) Springer Japan KK, part of Springer Nature 2017

\section{Introduction}

Human activity at multiple scales is the primary driver of the environmental challenges humanity faces (Steffen et al. 2007). Numerous scholars have argued that addressing environmental problems will require large-scale change in human behavior and the institutional, social and cultural forces that shape behavior (Princen 2003; Speth 2008; Beddoe et al. 2009; Assadourian 2010; Kinzig et al. 2013). In fact, most definitions of sustainability and sustainable development implicitly or explicitly recognize the need for changes in human perspectives, aspirations, technologies, norms, or worldviews-in short, culture ${ }^{1}$.

However, calls for cultural change often stop short of proposing the precise mechanisms through which such change may occur precisely because the relevant mechanisms of behavioral and cultural change are not known. The multiple disciplines that comprise the social sciences and humanities have different and, often competing, theories of cultural change that operate at multiple levels of human organization. These disciplinary differences have been a challenge for sustainability science (Gardner 2013), and the absence

Handled by Osamu Saito, UNU-Institute for the Advanced Study of Sustainability, Institute of Sustainability and Peace, Japan.

Jeremy S. Brooks

brooks.719@osu.edu

1 School of Environment and Natural Resources, Ohio State University, Columbus, OH, USA

2 Mitchell Center for Sustainability Solutions and School of Economics, University of Maine, Orono, ME, USA

3 Department of Anthropology, Graduate Group in Ecology and Center for Population Biology, University of California, Davis, USA

4 Department of Environmental Science and Policy, University of California, Davis, USA of a robust, non-disciplinary, theoretical framework hinders progress towards a deeper understanding of when and how sustainable social-ecological systems emerge (Levin and Clark 2010). Recently, sustainability scientists have been explicit about the need to incorporate mechanisms of cultural change in their research (Beddoe et al. 2009; Caldas et al. 2015) and to clarify the exact mechanisms involved (Ehrlich and Levin 2005; Waring et al. 2015). Importantly, cultural evolution theory offers an integrative approach to studying the dynamics of cultural change based on causal models of the mechanisms through which individual and population processes interact.

Despite many examples of sustainable resource management, exploitative and unsustainable resource management are common (Steffen et al. 2007). However, cultural change may be important for driving the proliferation of sustainable practices. This is because, although evolved genetic mechanisms, ecological processes, and socio-cultural mechanisms all influence resource use, social conditions often change more quickly than ecological conditions and cultural evolution is more rapid than genetic evolution (Perreault 2012). As such, there is an urgent need for sustainability scientists to develop more holistic or inclusive models to explain and integrate socio-cultural mechanisms of change at both individual and institutional levels (Borgerhoff Mulder and Coppolillo 2005). Such models are needed to inform sustainability policy solutions that can be applied cross-culturally and in divergent contexts. Currently, however, the dynamics of cultural change are not well understood in the context of sustainability. By focusing on applications of cultural evolution, we view this special issue as a starting point for determining how we can harness processes of cultural change

\footnotetext{
${ }^{1}$ Definitions of sustainability have multiplied in recent decades, but we see value in the flexibility with which the term can be employed (e.g. Kates et al. 2005). Importantly, several scholars (Gallopin 2003) view sustainable development as an ongoing and dynamic process, which also highlights the importance of understanding cultural change.
} 
(Wilson et al. 2014) to build more sustainable communities and societies.

The study of cultural evolution seeks to explain the diversity of human behavior observed worldwide and offers a mechanistic, causal framework for understanding changes in socially learned norms, values, and institutions (Richerson and Boyd 2005). It draws heavily from the diffusion of innovations literature (Richerson and Boyd 2005) and is complementarity with psychology (Mesoudi 2009), economics (van den Bergh and Gowdy 2000; Bowles 2004; Hodgson and Knudsen 2010; Safarzyńska 2013), and organizational theory (Hodgson 2013), among other frameworks (Gintis 2007). Cultural evolution gives us a unique view into the factors that shape the behaviors that ultimately contribute to, or hinder, the transition to a more sustainable society. Thus, there is value in synthesizing the theoretical (Boyd and Richerson 1985; Henrich 2004) and empirical literature (Campbell 1969; McElreath et al. 2005; Henrich et al. 2010) on cultural evolution with social-ecological systems research (Anderies et al. 2004; Janssen and Anderies 2007; Ostrom 2009) and sustainability science more broadly. This synthesis is especially timely given the recent confluence of cultural evolution models with multilevel selection theory (Wilson 1975; Wilson et al. 2013).

The cultural multilevel selection (CMLS) framework recognizes the potential for evolutionary pressures to operate on multiple scales simultaneously. This framework is particularly valuable because it has been used to explain the evolution of cooperation (Wilson 1975; Traulsen and Nowak 2006; Wilson and Wilson 2007), which is an almost ubiquitous challenge in the context of sustainability and sustainable resource use. CMLS and related models also help explain the social transmission of altruistic behavior (Wilson and Kniffin 1999), resource conservation under climate instability (Safarzynska 2013), the emergence of economic institutions (Bowles et al. 2003), and the evolution of sustainable resource management institutions (Waring et al. 2017). As such, we see cultural evolution as a tool that allows sustainability scientists to incorporate cultural dynamics into their work, especially the emergence and spread of sustainable behaviors, practices, norms, and institutions.

The goal of this special issue is to introduce readers to cultural evolution theory and its applications in sustainability science, thereby catalyzing future research in this field. The special issue emerged from a working group funded by the National Socio-Environmental Synthesis Center (SESYNC) in Annapolis, MD. The objective of this working group was to unite cultural evolution scholars with empirical social-ecological systems researchers to create an integrative evolutionary framework of social-ecological systems change. We sought to develop a clearer interdisciplinary articulation of the synthetic evolutionary framework, to reinterpret current and historical social-ecological case studies through the lens of cultural evolution theory, and to develop novel testable hypotheses about social-ecological systems change. Before describing the papers in this special feature, however, we briefly summarize cultural evolution theory and the cultural multilevel selection framework.

\section{A brief background on cultural evolution}

The idea that there are parallels between how cultural traits change and how biological species evolve dates back to Darwin (Darwin 1874). However, cultural evolution itself is a relatively new field. The foundational mathematical models on the topic were published in the1980s (Cavalli-Sforza and Feldman 1981; Boyd and Richerson 1985). In the more than 3 decades since there has been substantial growth in cultural evolution research, evidenced by the emergence of a new professional society, the Cultural Evolution Society, which held its first meeting in 2017. Several accessible review articles (Richerson and Henrich 2012; Mesoudi 2016), book chapters (Morgan et al. 2015), and books (Richerson and Boyd 2005; Mesoudi 2011; Henrich 2015; Boyd 2017) have covered the general principles, core concepts, and emerging insights in this field. We provide a brief overview to explain the relevance of cultural evolution for sustainability scientists.

Culture has many meanings that can depend, in part, on one's disciplinary background (Caldas et al. 2015). In the cultural evolution literature, "culture" is defined as socially transmitted information, which can include beliefs, values, behaviors, and knowledge, and-more specific to sustainability science-the technologies, lifestyles, consumption patterns, norms, institutions, and worldviews that ultimately shape human impacts on the environment. This approach typically focuses on individual cultural traits or variants (Boyd and Richerson 1985; Mesoudi 2016).

While culture entails socially rather than genetically transmitted information, change in the distribution of cultural traits in a human population can be viewed as a Darwinian process (Darwin 1874; Campbell 1960; Simonton 2010). This is because cultural change entails heritable variation and differential propagation through time. Individuals hold and express different beliefs, values, knowledge, and behaviors (variation) that can be observed by, shared with, and imitated by other individuals in a population (inheritance). Some traits spread through a population more than others as a direct function of their effects. That is, traits that are perceived to be associated with success, or to generally lead to favorable outcomes are more likely to be copied (payoff based transmission). In other cases, traits may be copied because they are more common in a population (conformitybiased transmission) or because they are expressed by prestigious individuals, even if those traits did not directly result 
in the high status of the individual modeling that behavior (prestige-biased transmission). Traits may also get copied more preferentially based on their content, regardless of the ultimate effect on an individual. For instance, experimental studies suggest that individuals are more attentive to cultural traits that generate positive or negative emotional reactions or contain social information (Mesoudi 2016) because such behaviors or beliefs were likely to have been adaptive in our ancestral environments (Richerson and Henrich 2012; Mesoudi 2016).

Another important force in cultural evolution is guided variation, in which individuals or groups create new traits by learning, research, and similar endeavors. In conjunction with the transmission biases outlined above, guided variation can lead to the rapid spread of new behaviors and institutions. Individuals and groups may consider multiple innovations before deciding on which one to adopt, which can itself be modeled as a Darwinian process (Campbell 1960; Simonton 2010). Research teams, legislative committees, panels of judges, constitutional conventions and many other kinds of groups can invent new variants. Thus, unlike in genetic evolution where variation arises only by random mutation, created cultural variation is usually not completely random with respect to outcomes. While group-level guided variation is not as well studied by cultural evolutionists as that at the individual level, it has been well studied in other research traditions such as political science (Sabatier and Jenkins-Smith 1993) and social psychology (Haslam 2001).

Guided variation is also a multi-level process. A common form of institutional evolution is to remodel institutions considered functional at a lower level to apply them to a higher level, which combines biased transmission and guided variation. For example, in federal systems a successful innovation at the provincial level may be modified as an innovation at the national level such as occurred in the United States with the adoption of federal policies from women's suffrage and same-sex marriage (Tribou and Collins 2015). Similarly, community-based natural resource management practiced in specific locations prompted a global trend in conservation interventions (Hulme and Murphree 1999). Likewise, global institutions can arise by creatively scaling up national institutions, as can be seen with the United Nations, International Monetary Fund, World Trade Organization, and the World Bank.

Guided variation and cultural transmission biases can then shape population-level dynamics and can be used to understand and anticipate cultural change over time. Any factor that changes the frequency of traits in a population contributes to evolutionary change. But forces with a selective influence on traits in the population, such as the natural selection of cultural traits on their outcomes and various selective social learning mechanisms, can contribute to cultural adaptation to the environment. Group-level cultural change is especially important in so far as sustainable resource use challenges often evoke social dilemmas that can typically be solved through intergroup dynamics, as we explore in more detail below.

\section{Group structures, multi-level evolutionary processes, and sustainability}

Humans are social animals that are well adapted to group social life. We are attentive to the symbols and characteristics that differentiate groups, respond to social norms and group conventions, and often prefer to interact with those with whom we share a common group identity (McElreath et al. 2003; Bernhard et al. 2006; Rusch 2014). These fundamental group-level cultural dynamics are important for understanding human behavior generally and also influence environmental cooperation.

Cooperation is an essential concept in sustainability science because many of the most pressing environmental challenges can be characterized as social dilemmas-situations in which the best outcomes for individuals conflicts with the best outcome for the group collectively. Cooperation sometimes requires individuals to bear personal costs to provide diffuse or long-term benefits to a broader group, for example to protect biodiversity for future generations. When cooperation entails net personal costs (i.e., altruism) freeriders who benefit from the cooperation of others without paying the costs will outcompete cooperators, which is not an evolutionarily stable strategy (Smith and Winterhalder 1992). For this reason, the evolution of cooperation has been a puzzle for biologists.

However, cooperation within groups can evolve when groups compete. Though the evolutionary fitness of a cooperator is lower than non-cooperators within a group, groups of cooperators may still outcompete groups of selfish individuals. When cooperative groups outcompete noncooperative groups the behaviors, norms, and institutions of the dominant group (including cooperative tendencies) can spread via a number of mechanisms (Boyd and Richerson 2002, 2009; Henrich 2004). The process of the adaptive change of group traits due to group competition is called group selection. Cooperation can also arise when groups play games against hostile nature as well as games against competing groups (Gavrilets and Richerson 2017).

Group selection forces are stronger when the trait at hand varies more between groups than within groups (Price 1972; Henrich 2004). Because natural populations are usually genetically well mixed, group selection is deemed rare in nature. Human groups, however, have greater grouplevel variation in culture than in genes (Bell et al. 2009) and cultural mechanisms like conformist transmission, ethnic marking, punishment of norm-violators, and normative 
conformity maintain distinct group identities and group-level variation. Thus, cultural group selection provides a plausible theoretical explanation for the evolution of cooperation in human societies (Henrich 2004).

A long history of cultural group selection has probably been responsible for humans' rather prosocial predispositions (Richerson et al. 2016). Culturally constructed environments act as selective factors on genes, as when institutions reward conformity and punish non-conformity. Humans are comparatively docile (Simon 1990), learn norms easily and conform to them (Chudek and Henrich 2011), and are sensitive to social reinforcement (Baum 2017). Darwin (1874) argued that prosocial "instincts" like patriotism and sympathy could form the basis for the cultural evolution of better institutions (Richards 1988). Humans tend to favor institutions that solve dilemmas of cooperation and our large-scale social systems are arguably a product of innate biases that are ultimately a product of cultural group selection.

The presence of group selection does not, however, mean that individual selection pressures disappear. Instead selection pressures occurring simultaneously at different levels must be balanced to gauge the overall effect of evolution. Multilevel selection theory helps to account for these simultaneous pressures (Okasha 2006). Within groups, cooperative individuals who bear the costs of providing group benefits will tend to have lower fitness relative to freeriding group members. However, their cooperation can nonetheless increase the fitness of their group relative to other groups, enabling cooperation to spread in the population over time. Institutionalized systems of reward and punishment can also align individual interests with group functions, reducing the impact of within-group selfishness. Moreover, the evolution of such institutional systems will tend to be favored by higher level selection processes. Therefore, the cultural multilevel selection framework (CMLS) asks when group selection pressures outweigh individual selection pressures, and uses that balance as the key indicator for the emergence of cooperation in environmental dilemmas.

\section{The conditions for cultural group selection}

The application of cultural evolution and multilevel selection to sustainability science generates a crucial hypothesis about the emergence of conservation behaviors and policies (Waring et al. 2015). The hypothesis is that cooperative environmental traits such as conservation behaviors and policies are more likely to spread when the dominant level of cultural selection is at or above the social scale of the relevant environmental dilemma. However, the CMLS framework is complex and requires careful application and attention to definitions. For example, detecting group-level cultural features, such as a recycling policy, is not equivalent to detecting group-level adaptations for environmental action. Kline et al. (this issue) carefully lay out the necessary and sufficient conditions for cultural group selection and grouplevel cultural adaptations.

In brief, cultural group selection (also "group-level cultural selection") requires three factors, (1) group-structured cultural traits (e.g., an institution or group-level variation in individual behavior, such as resource consumption) which generate, (2) group-structured outcomes that differentiate groups with and without the trait in significant ways, and (3) a mechanism of between group selection, such as extinction of groups or trait transmission between groups. With these conditions met, Kline et al. argue that cultural group selection occurs, but it may still not contribute to group-level cultural adaptations. For that, the strength of group-level cultural adaptation must overwhelm other evolutionary processes such as individual adaptation, or cultural drift. Kline et al.'s contribution clarifies the foundational mechanisms and concepts used throughout the rest of the special issue. We outline the key components and contributions of the remaining articles in the special issue below.

\section{A summary of papers in the issue}

The special issue includes three articles that originated in the SESYNC working group (Brooks et al.; Hillis, et al.; Kline et al.) and seven that were received from an open solicitation. Among other topics of importance for sustainability science, these ten papers include re-interpretations of classic case studies of long-standing resource management systems (Waring and Acheson; Brooks et al.), evaluations of historical policy change (Hanes and Waring), a prospective research design exercise (Andrews and Borgerhoff Mulder), new analyses based on prior data (Macfarlan and Remiker; Hillis et al.), and a methodological guide for designing and implementing new studies of cultural evolution in the context of social-ecological systems change (Kline et al.).

This set of papers illustrates how cultural evolution theory can contribute to sustainability science in several ways. While most papers focus on a single case or set of related cases, Ellis et al., take a longer and broader view of the evolution of human environmental use. These authors propose that most of the major human solutions for environmental management have evolved through group-level selection on human culture. Ellis et al. suggest that humanity's modification of the environment is not simply the result of aggregate individual action, but rather the consequence of human group actions and innovations that allowed groups to become more adept at exploiting their environment. The authors argue that the scale of human society has coevolved with the intensity of human ecosystem engineering in a reciprocal system of causation. 
Other papers in the special issue examine cultural group selection in the context of mobile (Brooks et al.) and (nearly) stationary (Acheson and Waring) natural resources, agricultural products (Hanes et al.; Hillis et al.; Macfarlan and Remiker), the political sustainability of activist campaigns (Frost), and challenges pertaining to developing global policies for climate change (Zefferman) and local dynamics related to climate change mitigation strategies (Andrews and Borgerhoff Mulder). Several papers are based on a retrospective application of the CMLS framework to examine the likelihood that cultural group selection resulted in the emergence and spread of a trait that facilitated sustainable resource management (Acheson and Waring; Brooks et al.; Hanes et al.). Importantly, two of these cases explicitly focused on classic cases studies of common pool resource management-irrigation water management in Bali and lobster fishing in Maine. These cases are important because (1) they are well known, and the familiar context may make it easier for readers to conceptualize the dynamics of cultural multilevel selection, and (2) they have been the object of intense study for long periods of time, which has produced rich ethnographic, historical and ecological data.

Other papers apply cultural evolution and/or CMLS to contemporary cases and analyze existing data in new ways. Macfarlan and Remiker offer a detailed study of cooperation within labor sharing groups in Dominica before, during, and after a trade recession. They use a longitudinal dataset to examine the degree to which labor exchange and social support networks were affected by a collapse of the bay oil market. Hillis et al. test preliminary hypotheses derived from the CMLS perspective by studying sustainable management practices adopted by wine grape growers in California. The authors describe how group structures like wineries and regional partnerships create regional brands and identities, operate certification programs, and provide outreach and education programs that can affect the spread of these practices. The authors use data from three regions to test the hypothesis that there is greater scope for group selection to act on sustainable management practices that are costly to individual farmers, thus embodying a cooperative dilemma, than for practices that are not perceived to be costly for farmers. Frost takes a unique approach using cultural evolution theory to examine the political sustainability of grassroots campaigns organized by First Nations communities in Canada against environmentally destructive industries. He characterizes political solidarity in direct action campaigns as a commons problem and argues that such grassroots campaigns implicitly use cultural evolution to adaptively manage their campaigns. Frost discusses the different social dynamics at play in large-group campaigns and small-group campaigns and describes the transmission of values and strategies within and between activist campaigns.
Still other papers are prospective in nature. For instance, Andrews and Borgerhoff Mulder explore the possibility for studying CMLS dynamics in real time. On Pemba Island (Zanzibar, Tanzania) individual and group level payoffs to forest conservation behavior are being influenced by the United Nations intervention for Reducing Emissions from Deforestation and Degradation (REDD+). Under REDD + the institutionalized systems of rewards and punishments align individual interests with those of the group. The authors suggest that given this intervention, sustainable forestry institutions and practices can evolve by enhancing the covariance between individual success and community success. Andrews and Borgerhoff Mulder describe how the design of REDD+ matches a CMLS approach to generating cooperative forest conservation among forest users, and illustrate that some sustainability interventions, even large ones like REDD+, can work on principles that match the CMLS approach very well. At a different scale, Zefferman constructs an argument about the likelihood of the emergence of planetary scale cooperation in carbon emissions reduction and climate change. He suggests that because there is no strong force of cultural selection or competition acting on the level of the planet (such as competition with Mars for a livable atmosphere might create), it is unlikely that cooperation at the global scale will evolve to reduce global carbon emissions voluntarily.

Importantly, several authors aim to illustrate how cultural evolution and CMLS complement existing frameworks for understanding cultural change and sustainability. For instance, Macfarlan and Remiker suggest that CMLS and Biological Market Theory (which emphasizes variability in potential partners and the ability to choose partners) are both needed to fully explain the dynamics of labor exchanges and social support networks. Brooks et al. use CMLS to examine the emergence and spread of synchronized cropping and the institutions, called subaks, that support such coordination in Bali. Their work draws heavily on the historical, anthropological, and archaeological research produced by Steven Lansing and colleagues who suggest that Balinese subaks constitute a complex adaptive system (CAS). Brooks et al. suggest that their evolutionary explanation builds on and complements the CAS perspective by describing the selection pressures that could have given rise to increasingly complex institutional structures. In addition, Hillis et al., and Andrews and Borgerhoff Mulder highlight the foundational role that the diffusion of innovations literature has played in the development of cultural evolution, but indicate that the CMLS framework adds additional explanatory value for understanding the spread of traits that entail cooperative dilemmas or involve tradeoffs at different levels of social organization. Finally, Frost's description of the social dynamics of grassroots activism among indigenous communities in Canada describes how cultural evolution aligns 
nicely with Ostrom's social-ecological systems framework, Whitehouse's theory of dual modes of religiosity, and Atran's work on the psychology of the sacred.

\section{Important insights and implications}

Several key findings from this issue will be of interest to sustainability scientists. One finding pertains to the potential role of group selection in the emergence and spread of group territoriality (as demonstrated in the analysis of sustainable lobster fishing, Waring \& Acheson). A second concerns how changes in the dominant level of selection and cultural group selection mechanisms may explain complex institutional structures (such as the rites and rituals associated with the agro-ecological systems in Bali, Brooks et al.). Both of these results have implications for our understanding of common pool resource management (Ostrom 1990).

In direct reference to Waring et al.'s (2015) hypothesis, several other papers (e.g. Hanes and Waring; Brooks et al.; Macfarlan and Remiker; Andrews and Borgerhoff Mulder) also note the importance of considering the dominant level of selection relative to the scale of the environmental dilemma in question and note potential changes in the dominant level of selection over time. For example, Hanes and Waring use their detailed historical study of the blueberry industry in Maine to argue that the concept of the dominant level of selection helps to explain how matches and mismatches between resource scale and institutional scale arise. They find that the establishment of a research station funded by a self-imposed tax on industry can plausibly be explained as the result of severe economic selection pressure and could represent an instance of institutional evolution via cultural group selection. In addition, Macfarlan and Remiker suggest that changes in bay oil regulations and a subsequent collapse in the international market for bay oil ultimately shifted the dominant level of selection in a way that negatively affected cooperation in labor exchanges and resulted in a decline in social support networks.

A number of papers also have clear practical implications for contemporary environmental challenges. In their prospective paper on REDD + in Tanzania, Andrews and Borgerhoff Mulder show how thinking about REDD + within the CMLS framework yields new insights for field application. First, they note the potential for comparing the effectiveness of payments to individuals (as cash) and payments to communities (as social services or infrastructure) in promoting new forest conservation norms. Second, they observe how a commonly recognized institutional weakness of REDD+ could generate intergroup dynamics favorable to a cultural multilevel selection cascade (Waring et al. 2015). "Leakage" occurs when individuals in protected forestry areas turn their harvest pressure to unprotected neighboring forests, thereby reaping the carbon credit payments and at the same time externalizing their individual costs. The authors discuss how, counterintuitively, leakage might accelerate the spread of conservation practices between groups insofar as unprotected groups may seek protection from predatory neighbors through entering the REDD+ program.

In their study of sustainable viticulture practices, Hillis et al. find that, consistent with hypotheses derived from the CMLS framework, the fraction of between-group variation is greater for practices that are cooperative dilemmas than for non-cooperative practices. This result suggests that there is greater scope for group selection for cooperative practices relative to non-cooperative practices. The authors also outline and provide evidence for several mechanisms through which group selection might spread sustainable management practices including competition between regions, imitation of successful groups, and differential proliferation of groups. This work has important implications for agricultural industries in which regional branding and other group level structures can potentially shape the behaviors of individual farmers and provide a mechanism for the spread of more sustainable practices.

In addition, Frost finds that the transmission of sacred values in the process of recruitment, along with the ability of small-groups to generate tight social bonds and high levels of solidarity, can be critical for the sustainability of grassroots campaigns. He concludes that it is important for groups to be engaged in adaptively managing the dynamics of social transmission and the social learning process that can occur between campaigns.

Zefferman suggests that current models of the emergence of global solutions to global environmental challenges are flawed. His paper leaves an open question for the field: are global cooperative solutions possible, or is the logic of CMLS iron-clad? One possibility is that CMLS has paid inadequate attention to the force of collective guided variation that operates to some extent at the global level, for example in negotiating the Paris Climate Accord. Such processes, insofar as they lead to collective international agreements, could generate effective solutions to commons problems, including global ones. The CMLS framework highlights that, without a system of rewards and punishments that align countries' goals with all countries collective interest in the global commons, we have to worry that country defections will undermine such voluntary accords.

Similar to Zefferman, most authors conclude by asking provocative questions or providing insights for future research on sustainability challenges. For instance, Ellis et al. pose what may be a central question for sustainability science: if sociocultural niche construction via cultural group selection is the mechanism most directly responsible for the current sustainability crisis, can it plausibly also guide positive cultural and institutional change towards 
sustainable solutions? Kline et al. anticipate the needs of future researchers and explain how to gather and evaluate evidence to confirm or reject the hypothesis that a given group-level cultural trait is an adaptation. This approach allows researchers to understand whether a group is succeeding for reasons independent of the group level traits that helped them solve an environmental dilemma, thus avoiding the trap of adaptationist thinking. Kline et al. provide additional tools to help researchers achieve higher quality CMLS analyses, including a decision map for building CMLS hypotheses and an R script for quantitative testing of group structure in empirical datasets.

\section{Conclusion}

Our endeavor in this special feature is to apply cultural evolution and the CMLS framework to sustainability science, social-ecological systems research, and environmental challenges more broadly. Our more specific objectives are to outline a robust theoretical framework for understanding how sustainable social-ecological systems might emerge, to delineate the kinds of data needed to test such frameworks, and to explore their potential in explaining the patterning of sustainable behaviors across time and space in real socioecological systems, past and present. Because the use of cultural evolutionary models in the domain of sustainability is still so new, most of the papers in this special issue are based on analyses that are retrospective, using data that were inevitably collected with other frameworks in mind. Indeed, an important challenge for applications of cultural evolution and CMLS is that these are 'data hungry' frameworks, as outlined in Kline et al. That said, these papers represent important initial steps in refining the application of these cultural evolutionary models for understanding whether and in which contexts sustainable social-ecological systems can emerge and persist. Taken together, they demonstrate exciting applications of evolutionary thinking to topics and questions of interest to sustainability scientists and social-ecological systems researchers and point the way for future research that can help both solidify theory and guide practical approaches to solving sustainability challenges.

Acknowledgements This work was supported by the National SocioEnvironmental Synthesis Center (SESYNC) under funding received from the National Science Foundation DBI-1052875 and the Ohio Agricultural Research and Development Center.

\section{References}

Anderies J, Janssen M, Ostrom E (2004) A framework to analyze the robustness of social-ecological systems from an institutional perspective. Ecol Soc 9:1-17
Assadourian E (2010) The rise and fall of consumer cultures. In: State of the World 2010: transforming cultures. W. W. Norton and Co., New York, pp 3-20

Baum WM (2017) Understanding behaviorism: behavior, culture, and evolution. Blackwell Publishing, Malden, MA

Beddoe R, Costanza R, Farley J et al (2009) Overcoming systemic roadblocks to sustainability: the evolutionary redesign of worldviews, institutions, and technologies. Proc Natl Acad Sci 106:2483-2489. https://doi.org/10.1073/pnas.0812570106

Bell AV, Richerson PJ, McElreath R (2009) Culture rather than genes provides greater scope for the evolution of large-scale human prosociality. Proc Natl Acad Sci 106:17671

Bernhard H, Fischbacher U, Fehr E (2006) Parochial altruism in humans. Nature 442:912-915

Borgerhoff Mulder M, Coppolillo P (2005) Conservation: linking ecology, economics and culture. Princeton University Press, Princeton

Bowles S (2004) Microeconomics: behavior, institutions, and evolution. Princeton University Press, Princeton

Bowles S, Choi J-K, Hopfensitz A (2003) The co-evolution of individual behaviors and social institutions. J Theor Biol 223:135-147

Boyd R (2017) A different kind of animal: how culture transformed our species: how culture transformed our species. Princeton University Press, Princeton

Boyd R, Richerson PJ (1985) Culture and the evolutionary process. University of Chicago Press, Chicago

Boyd R, Richerson PJ (2002) Group beneficial norms can spread rapidly in a structured population. J Theor Biol 215:287-296

Boyd R, Richerson PJ (2009) Culture and the evolution of cooperation. Philos Trans R Soc B Biol Sci 364:3281-3288

Caldas MM, Sanderson MR, Mather M et al (2015) Endogenizing culture in sustainability science research and policy. Proc Natl Acad Sci 112:8157-8159

Campbell DT (1960) Blind variation and selective retentions in creative thought as in other knowledge processes. Psychol Rev 67:380

Campbell DT (1969) Variation and selective retention in socio-cultural evolution. Gener Syst 14:69-85

Cavalli-Sforza LL, Feldman MW (1981) Cultural transmission and evolution: a quantitative approach. Princeton University Press, Princeton

Chudek M, Henrich J (2011) Culture-gene coevolution, norm-psychology and the emergence of human prosociality. Trends Cogn Sci 15:218-226

Darwin C (1874) The descent of man, revised edition. The Henneberry Company, Chicago

Ehrlich PR, Levin S (2005) The evolution of norms. PLOS Biol 3:943-948

Gallopin G (2003) A systems approach to sustainability and sustainable development. Sustainable Development and Human Settlements Division, United Nations, Santiago

Gardner SK (2013) Paradigmatic differences, power, and status: a qualitative investigation of faculty in one interdisciplinary research collaboration on sustainability science. Sustain Sci 8:241-252

Gavrilets S, Richerson PJ (2017) Collective action and the evolution of social norm internalization. Proc Natl Acad Sci 114(23):6068-6073

Gintis H (2007) A framework for the unification of the behavioral sciences. Behav Brain Sci 30:1-16. https://doi.org/10.1017/ S0140525X07000581

Haslam SA (2001) Psychology in organizations: the social identity approach. SAGE Publications Ltd., London

Henrich J (2004) Cultural group selection, coevolutionary processes and large-scale cooperation. J Econ Behav Organ 53:3-35

Henrich J (2015) The secret of our success: how culture is driving human evolution, domesticating our species, and making us smarter. Princeton University Press, Princeton 
Henrich J et al (2010) Markets, religion, community size and the evolution of fairness and punishment. Science 327:1480-1484

Hodgson GM (2013) Understanding organizational evolution: toward a research agenda using generalized darwinism. Org Stud 34:973992. https://doi.org/10.1177/0170840613485855

Hodgson GM, Knudsen T (2010) darwin's conjecture: the search for general principles of social and economic evolution. University Of Chicago Press, Chicago

Hulme D, Murphree M (1999) Communities, wildlife, and the "new conservation" in Africa. J Int Dev 11:277-285

Janssen MA, Anderies JM (2007) Robustness trade-offs in socialecological systems. Int J Commons 1:43

Kates RW, Parris T, Leiserowitz AA (2005) What is sustainable development? Goals indicators, values and practice. Environ Sci Policy Sustain Dev 47:8-21

Kinzig A, Ehrlich PR, Alston LJ et al (2013) Social norms and global environmental challenges: the complex interaction of behaviors, values, and policy. Bioscience 63:164-175

Levin SA, Clark WC (2010) Toward a science of sustainability. In: toward a science of sustainability. The National Science Foundation, Airlie Center-Warrenton, p 109

McElreath R, Boyd R, Richerson P (2003) Shared norms and the evolution of ethnic markers. Curr Anthropol 44:122-130

McElreath R, Lubell M, Richerson PJ et al (2005) Applying evolutionary models to the laboratory study of social learning. Evol Hum Behav 26:483-508

Mesoudi A (2009) How cultural evolutionary theory can inform social psychology and vice versa. Psychol Rev 116:929-952. https://doi. org/10.1037/a0017062

Mesoudi A (2011) Cultural evolution: how Darwinian theory can explain human culture and synthesize the social sciences. University of Chicago Press, Chicago

Mesoudi A (2016) Cultural evolution: a review of theory, findings and controversies. Evol Biol 43:481-497. https://doi.org/10.1007/ s11692-015-9320-0

Morgan TJ, Cross CP, Rendell LE (2015) Nothing in human behavior makes sense except in the light of culture: shared interests of social psychology and cultural evolution. Evolutionary perspectives on social psychology. Springer, New York, pp 215-228

Okasha S (2006) Evolution and the Levels of Selection. Clarendon Press Oxford, UK

Ostrom E (2009) A general framework for analyzing sustainability of social-ecological systems. Science 325:419-422

Perreault C (2012) The pace of cultural evolution. PLoS One 7:e4150

Price GR (1972) Extension of covariance selection mathematics. Ann Hum Genet 35:485-490

Princen T (2003) Principles for sustainability: from cooperation and efficiency to sufficiency. Glob Environ Polit 3:33-50

Richards RJ (1988) The Moral foundation of the idea of evolutionary progress: Darwin, Spencer, and the Neo-Darwinians. In: Nitecki MH (ed) Evolutionary progress. University of Chicago Press, Chicago, pp 129-148

Richerson PJ, Boyd R (2005) Not by genes alone: how culture transformed human evolution. University of Chicago Press, Chicago

Richerson PJ, Henrich J (2012) Tribal social instincts and the cultural evolution of institutions to solve collective action problems. Cliodynamics 3:38-80
Richerson P, Baldini R, Bell A et al (2016) Cultural group selection plays an essential role in explaining human cooperation: a sketch of the evidence. Behav Brain Sci 39:1-68. https://doi.org/10.1017/ S0140525X1400106X

Rusch H (2014) The evolutionary interplay of intergroup conflict and altruism in humans: a review of parochial altruism theory and prospects for its extension. Proc R Soc Lond B Biol Sci 281:20141539

Sabatier PA, Jenkins-Smith HA (1993) Policy change and learning: an advocacy coalition approach. Westview Press, Boulder

Safarzynska K (2013) The coevolution of culture and environment. J Theor Biol 322:46-57

Safarzyńska K (2013) Evolutionary-economic policies for sustainable consumption. Ecol Econ 90:187-195. https://doi.org/10.1016/j. ecolecon.2013.03.020

Simon H (1990) A mechanism for the social selection of successful altruism. Science 250:1665-1668

Simonton DK (2010) Creative thought as blind-variation and selectiveretention: Combinatorial models of exceptional creativity. Phys Life Rev 7:156-179

Smith EA, Winterhalder B (eds) (1992) Evolutionary ecology and human behavior. Aldine de Gruyter, New York

Speth JG (2008) The Bridge at the End of the World: Capitalism, the Environment, and Crossing from Crisis to Sustainability. Yale University Press, New Haven

Steffen W, Crutzen PJ, McNeill JR (2007) The anthropocene: are humans now overwhelming the great forces of nature. AMBIO A J Hum Environ 36:614-621. https://doi. org/10.1579/0044-7447(2007)36[614:taahno]2.0.co;2

Traulsen A, Nowak MA (2006) Evolution of cooperation by multilevel selection. Proc Natl Acad Sci 103:10952-10955

Tribou A, Collins K (2015) This Is How Fast America Changes Its Mind. In: Bloomberg.com. http://www.bloomberg.com/ graphics/2015-pace-of-social-change/. Accessed 4 Aug 2015

van den Bergh JC, Gowdy JM (2000) Evolutionary Theories in environmental and resource economics: approaches and applications. Environ Res Econ 17:37-57

Waring T, Kline MA, Brooks JS et al (2015) A multilevel evolutionary framework for sustainability analysis. Ecol Soc 20:1-34

Waring TM, Goff SH, Smaldino PE (2017) The coevolution of economic institutions and sustainable consumption via cultural group selection. Ecol Econ 131:524-532

Wilson DS (1975) A theory of group selection. Proc Natl Acad Sci 72:143-146

Wilson DS, Kniffin KM (1999) Multilevel selection and the social transmission of behavior. Human Nature 10:291-310

Wilson DS, Wilson EO (2007) Rethinking the theoretical foundation of sociobiology. Q Rev Biol 82:327-348

Wilson DS, Ostrom E, Cox ME (2013) Generalizing the core design principles for the efficacy of groups. J Econ Behav Organ 90S:S21-S32

Wilson DS, Hayes S, Biglan A, Embry D (2014) Evolving the fture: toward a science of intentional change. Behav Brain Sci $37: 395-460$ 